\title{
Optic nerve ultrasound for fluid status assessment in patients with severe preeclampsia
}

\author{
Gabrijela Brzan Simenc ${ }^{1}$, Jana Ambrozic ${ }^{2}$, Katja Prokselj², Natasa Tul ${ }^{1}$, Marta Cvijic², \\ Tomislav Mirkovic ${ }^{3}$, Helmut Karl Lackner ${ }^{4}$, Miha Lucovnik ${ }^{1}$

\footnotetext{
1 Department of Perinatology, Division of Obstetrics and Gynecology, University Medical Center Ljubljana, Slovenia

2 Department of Cardiology, University Medical Center Ljubljana, Slovenia

${ }^{3}$ Department of Anesthesiology and Intensive Therapy, University Medical Center Ljubljana, Slovenia

${ }^{4}$ Otto Loewi Research Center, Section of Physiology, Medical University of Graz, Austria
}

Radiol Oncol 2018; 52(4): 377-382.

Received 9 August 2018

Accepted 2 November 2018

Correspondence to: Miha Lucovnik, M.D., Ph.D., Division of Obstetrics and Gynecology, University Medical Centre Ljubljana, Šlajmerjeva 4, SI-1000 Ljubljana, Slovenia. Phone: +386 31318 681; Fax: +386 143975 90; E-mail: miha.lucovnik@kclj.si

Disclosure: No potential conflicts of interest were disclosed.

Background. There are no data on usefulness of optic nerve sheath diameter (ONSD) as a marker of patient's fluid status in preeclampsia. The objective was to examine potential correlation between ONSD and lung ultrasound estimates of extravascular lung water in severe preeclampsia.

Patients and methods. Thirty patients with severe preeclampsia were included. Optic and lung ultrasound were performed within 24 hours from delivery. ONSD was measured $3 \mathrm{~mm}$ behind the globe. Lung ultrasound Echo Comet Score (ECS) was obtained summing B-lines ("comet tails") in parasternal intercostal spaces bilaterally. Pearson's correlation analysis was used to assess the relationship between ONSD and ECS ( $p<0.05$ significant).

Results. Median ONSD was $5.7 \mathrm{~mm}$ (range 3.8-7.5 mm). Median ECS value was 19 (range 0-24). Statistically significant correlation was found between ONSD and ECS $\left(r^{2}=0.464 ; p<0.001\right)$.

Conclusions. Significant correlation between ONSD and ECS suggests optic ultrasound could be used for assessing fluid status and guiding peripartum fluid therapy in patients with severe preeclampsia.

Key words: preeclampsia; fluid status; ocular ultrasound; optic nerve sheath diameter; lung ultrasound; comet tail (B-lines) sign

\section{Introduction}

Preeclampsia, a multisystem disorder characterized by new-onset hypertension and either proteinuria or end-organ dysfunction after 20 weeks of gestation, affects 2 to $5 \%$ of pregnancies. ${ }^{1-4}$ The ability to assess fluid status is fundamental for optimal management of preeclamptic patients. Insufficient intravascular volume results in decreased oxygen delivery to tissues and exacerbates organ dysfunction. ${ }^{5,6}$ On the other hand, fluid excesses can lead to tissue edema due to extravascular fluid accumulation, which is especially pronounced in preeclampsia because of altered endothelial function causing increased capillary permeability., ${ }^{5,-9}$ The Confidential Enquiry into Maternal Deaths in the United Kingdom reported six deaths between 1994 and 1996 due to adult respiratory distress syndrome that appeared to be related to poor fluid management in women with preeclampsia. ${ }^{10}$ On the basis of this report, recommendations on limiting intravenous fluids to not more than to $80 \mathrm{ml} /$ hour or $1 \mathrm{ml} / \mathrm{kg} /$ hour have been made. ${ }^{11}$ Higher rates may, however, be necessary in some preeclamptic patients to adequately correct tissue hypoperfusion. ${ }^{12,13}$ A quick, non-invasive, bedside 
test to assess fluid status of patients with preeclampsia would, therefore, be very helpful to clinicians working in obstetric units.

Ultrasound measurement of optic nerve sheath diameter (ONSD) has been described as a simple and reliable means of determining increased intracranial pressure due to cerebral edema in nonpregnant critically ill patients. ${ }^{14,15}$ Signs of cerebral edema have been reported on magnetic resonance imaging in $71 \%$ to $100 \%$ of patients with preeclampsia and ONSD has recently been described to be increased in these patients. ${ }^{16-20}$ It is not clear, however, whether increased ONSD can be used as a marker of systemic tissue edema and fluid overload in this disease. The concern is that once dilated, ONSD could stay in pathologic values for several days. Therefore, more data on correlation between ONSD and other markers of fluid status are needed before ONSD measurements can be recommended as a guide to peripartum fluid management in preeclampsia.

Studies by our group and others have demonstrated that severe preeclampsia is associated with an increase in extravascular lung water (EVLW), which can be identified by lung ultrasound before appearance of clinical signs of pulmonary edema. ${ }^{13,21}$ The aim of the present study was to examine potential correlation between ONSD values and lung ultrasound estimates of EVLW in patients with severe preeclampsia.

\section{Patients and methods}

This prospective, observational study was performed at a single tertiary perinatal center from April 2015 to April 2017. Research was conducted following the Helsinki Declaration. All women included in the study provided written informed consent for study participation. The National Medical Ethics Committee approved the study (Project number 83/09/14, approved on 09/16/2014).

\section{Study participants}

Consecutively admitted patients with singleton pregnancies complicated by severe preeclampsia were included in the study at hospital admission. Severe preeclampsia was defined by severe features of preeclampsia using the American College of Obstetricians and Gynecologist Task Force on Hypertension in Pregnancy recommendations: new-onset cerebral or visual disturbances; pulmonary oedema; thrombocytopenia (platelet count
$<$ 100,000 /microliter); elevated liver enzymes (transaminases) to twice the normal upper limit, severe persistent pain in the right upper or middle upper abdomen that does not respond to medication and is not explained by another condition or both; renal insufficiency (serum creatinine $>97 \mu \mathrm{mol} / \mathrm{L}$ ), or a doubling of serum creatinine in the absence of other renal disease; systolic blood pressure $\geq 160$ $\mathrm{mm} \mathrm{Hg}$ or a diastolic blood pressure $\geq 110 \mathrm{~mm}$ $\mathrm{Hg}$ measured on more than one occasion at least 4 hours apart while the patient is on bed rest (unless antihypertensive therapy was initiated before this time). ${ }^{22}$ As per our institution's standard protocol all patients were managed in a high dependency setting antepartum and at least 24 hours post-delivery. Blood pressure was monitored continuously. Fluid intake and urine output were assessed hourly, and blood tests were repeated at least every 12 hours to monitor kidney function, electrolytes, full blood count, transaminases, and bilirubin. Magnesium sulphate was used for eclampsia prophylaxis as a $4 \mathrm{~g}$ intravenous loading dose, followed by $1 \mathrm{~g} /$ hour infusion. Antihypertensive treatment with intravenous hydralazine or labetalol was used to maintain systolic blood pressure at $<160 \mathrm{mmHg}$ and diastolic blood pressure at $<110 \mathrm{mmHg}$. Intravenous and oral fluid intake was minimized, and neither fluids nor diuretics were routinely administered to treat oliguria.

\section{Optic nerve sheath diameter (ONSD) and extravascular lung water (EVLW) measurements}

Ultrasound examination was performed using Vivid S6 scanner (GE Vingmed Ultrasound) within 24 hours from delivery. A 8L-RS linear probe was used for ONSD measurements and a 3Sc-RC cardiac probe for EVLW measurements.

ONSD measurements were performed in supine patients according to previously published protocol. ${ }^{14,15,19,23,24}$ Ultrasound gel was applied over the closed upper eyelid. Ultrasound probe was placed on the temporal area of the eyelid. The probe was then angled in order to display the entry of the optic nerve into the globe. ONSD was measured 3 $\mathrm{mm}$ behind the globe in the transverse plane perpendicular to the optic nerve. For each optic nerve three measurements were made. The reported ONSD corresponds to the mean of the six values obtained for each patient (three measurements for each eye). Three $\mathrm{mm}$ behind the ocular globe, the optic nerve sheath is surrounded only by orbital fat and can distend in cases of raised intracranial pres- 
sure due to cerebral oedema. ONSD of $>5.8 \mathrm{~mm}$ has been shown to detect increased intracranial pressure with a sensitivity of $90 \%$ and a specificity of $84 \%{ }^{25}$ Figure 1 presents an optic ultrasound image of one of the patients with severe preeclampsia included in the study. An increased ONSD can be clearly seen (Figure 1).

Lung ultrasound was performed according to a systematic protocol in supine patients. ${ }^{26,27}$ Increased amount of EVLW can be diagnosed by multiple B-lines or "comet tails" (Figure 2). ${ }^{28}$ B-lines are discrete laser-like vertical hyperechoic reverberation artefacts that arise from the pleural line and extend to the bottom of the screen without fading, and move synchronously with lung sliding. ${ }^{28}$ They represent a reverberation artefact through oedematous interlobular septa or alveoli. ${ }^{26,28}$ The sum of B-lines found in four areas in the parasternal line on the right (from the second to the fifth intercostal space), and in three areas in the parasternal line on the left (from the second to the fourth intercostal space) yielded the Echo Comet Score (ECS) denoting the amount of EVLW.

Inter-observer and intra-observer reproducibility for ONSD and EVLW measurements was validated at offline analyses in 10 randomly selected subjects (five preeclamptic patients and five controls) by two independent operators. The second operator was blinded to the patient's status (preeclampsia with severe features or control group).

\section{Statistical analysis}

Intra-class correlation coefficient (ICC) was used to test inter-observer and intra-observer reproducibility of the analysed parameters. Pearson's correlation analysis was used to assess the relationship between ONSD and ECS measurements. A twotailed $\mathrm{p}$ value $<0.05$ was considered statistically significant. The software used for statistical analysis was IBM SPSS Statistics for Windows Version 21.0 (Armonk, NY: IBM Corp.).

\section{Results}

Thirty severe preeclamptic patients were included in the study. Maternal race was Caucasian in all but one case of an Asian woman. None of the women included admitted to tobacco smoking, alcohol or drug abuse during pregnancy. None of the participants had chronic hypertension, pre-existent or gestational diabetes mellitus. None had prior ocular surgery or ocular trauma. Median maternal age

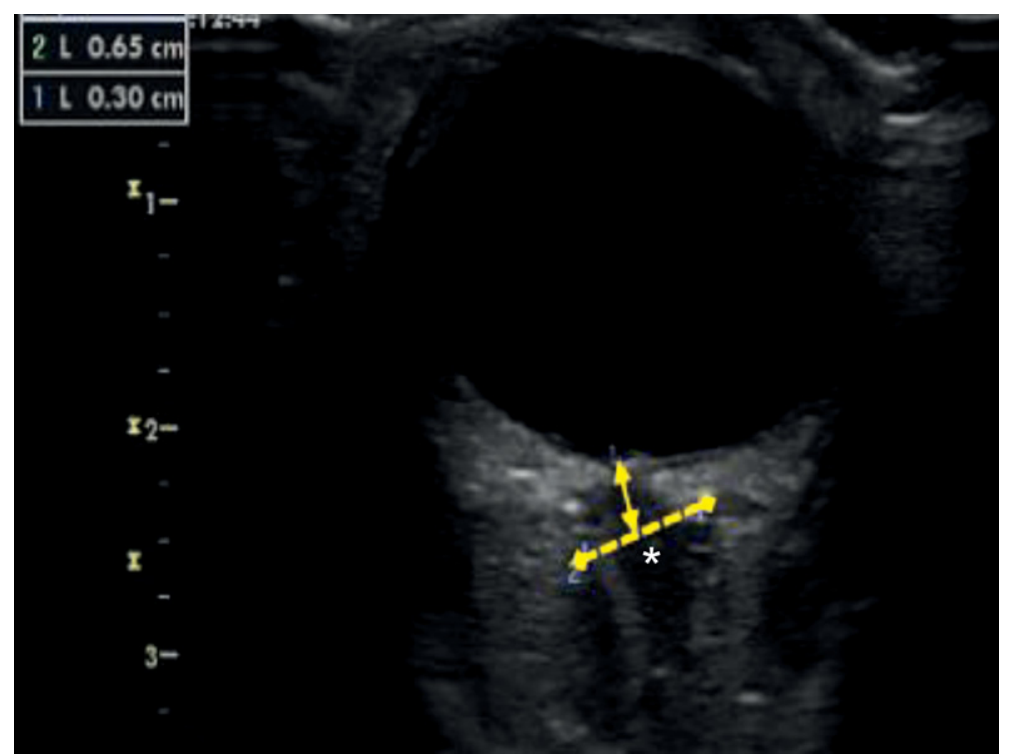

FIGURE 1. Increased optic nerve sheath diameter in one of the patients with severe preeclampsia included in the study $(*)$.

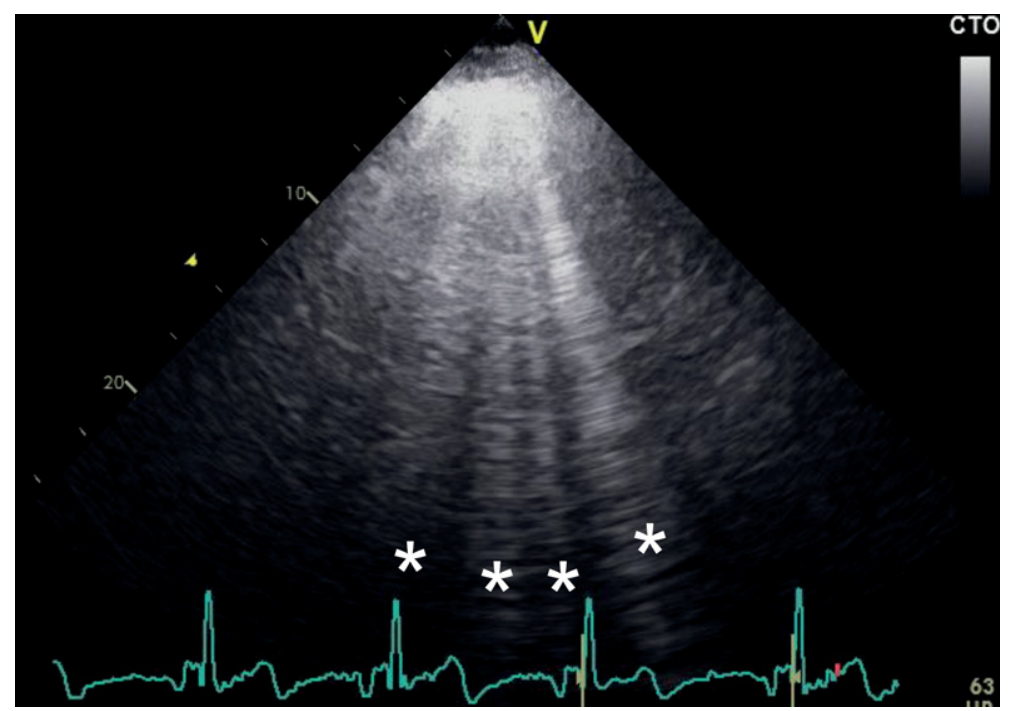

FIGURE 2. B-lines or "comet tails" (*) arising from the pleural line and spreading up to the edge of the screen representing excess of extravascular lung water in one of the patients with severe preeclampsia included in the study.

was 31 years (range 21-44 years); median maternal pre-pregnancy body mass index was $23 \mathrm{~kg} / \mathrm{m}^{2}$ (range 19-32 kg/m²), and median gestational age at study inclusion was 32 5/7 weeks (range 22 3/7-39 $4 / 7$ weeks). Twenty-three (77\%) patients were nulliparous. Severe features of preeclampsia meeting the inclusion criteria were: hypertension in all 30 cases, headache in 14 (47\%) cases, visual disturbances in four $(13 \%)$ cases, elevated liver enzymes in $10(33 \%)$ cases, thrombocytopenia in three (10\%) 
TABLE 1. Median and range of B-lines in four parasternal intercostal areas on the right (from the second to the fifth intercostal space) and three parasternal intercostal areas on the left (from the second to the fourth intercostal space)

\begin{tabular}{lcc}
\hline Parasternal area & Median number of B-lines & Range \\
\hline $2^{\text {nd }}$ intercostal space right & 0 & $0-3$ \\
$3^{\text {rd }}$ intercostal space right & 2 & $0-6$ \\
$4^{\text {th }}$ intercostal space right & 4 & $1-4$ \\
$5^{\text {th }}$ intercostal space right & 3 & $1-7$ \\
$2^{\text {nd }}$ intercostal space left & 3 & $0-6$ \\
$3^{\text {rd }}$ intercostal space left & 2 & $0-8$ \\
$4^{\text {th }}$ intercostal space left & 3 & $0-6$ \\
\hline
\end{tabular}

cases, and right upper abdomen pain in five $(17 \%)$ cases.

All 30 patients were treated with magnesium sulphate as per our institution's protocol. Magnesium sulphate was being administered as a continuous intravenous infusion of $1 \mathrm{~g} /$ hour (in a $50 \mathrm{ml} /$ hour infusion) during all ultrasound examinations. In $24(80 \%)$ patients, magnesium sulphate infusion was the only intravenous fluid intake. In six $(20 \%)$ patients, additional $30 \mathrm{ml} /$ hour infusion of crystalloids was being administered (always limiting intravenous fluid administration to $80 \mathrm{ml} /$ hour). Intravenous hydralazine was necessary for blood pressure control in six (20\%) patients.

Median ONSD value was $5.7 \mathrm{~mm}$ (inter-quartile range $5.2-6.0 \mathrm{~mm}$; range $3.8-7.5 \mathrm{~mm}$ ). Thirteen

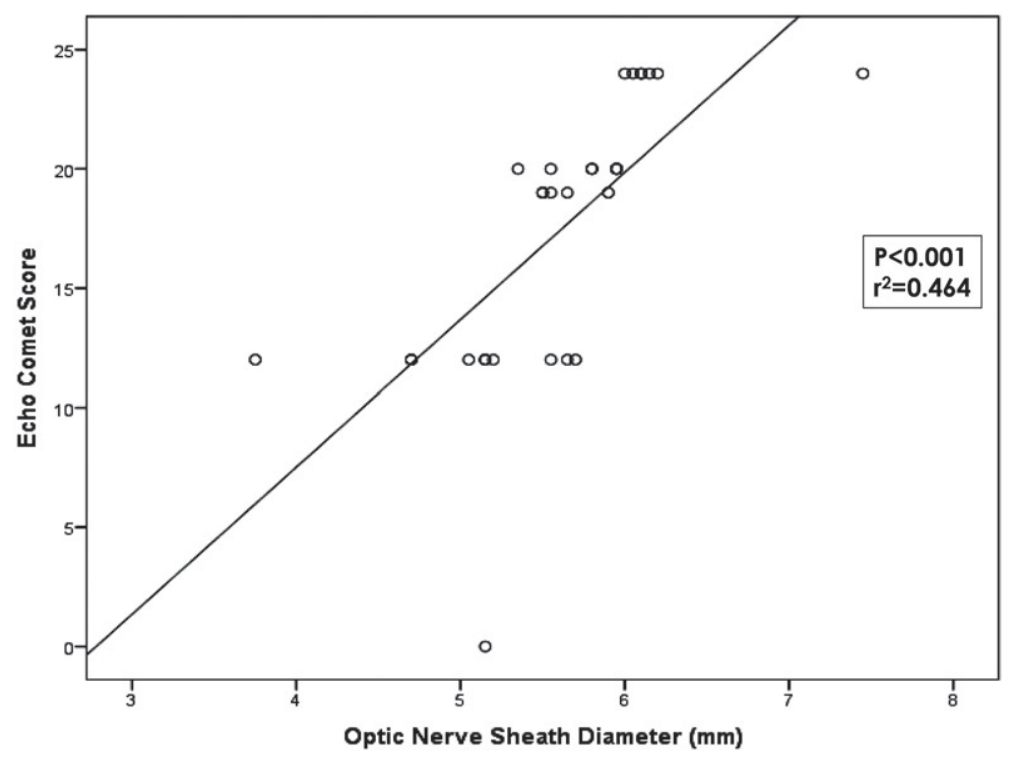

FIGURE 3. Correlation between optic nerve sheath diameter and lung ultrasound Echo Comet Score
(43\%) patients had ONSD > $5.8 \mathrm{~mm}$. Median ECS value was 19 (range $0-24$ ). Table 1 presents median and range values of B-lines in all areas analyzed.

Intra- and inter-observer agreement was excellent for ONSD measurements (ICC 0.973 [0.8900.993] and ICC 0.960 [0.839-0.990], respectively). Similarly, intra- and inter-observer agreement was also excellent for EVLW measurements (ICC 0.968 [0.915-0.987] and ICC 0.898 [0.846-0.993], respectively).

Statistically significant correlation was found between ONSD and ECS values $\left(\mathrm{r}^{2}=0.464 ; \mathrm{p}<\right.$ 0.001) (Figure 3).

\section{Discussion}

The main finding of our study is that ONSD correlates significantly with the amount of EVLW in severe preeclampsia. A dilated optic nerve, therefore, indicates systemic edema and fluid overload in patients with this condition.

The majority of studies on ONSD have been performed in traumatic brain injury patients. ${ }^{14,23,29,30}$ These studies demonstrated that ONSD correlates well with invasive measurements of intracranial pressure. ${ }^{14,15}$ Optic ultrasound has also been found to be a useful diagnostic tool for detecting intracranial hypertension in patients with meningitis, stroke, hepatic encephalopathy, epilepsy, and mountain sickness. ${ }^{31-35}$ Dubost et al. and our group have recently reported increased ONSD values in preeclamptic patients compared to healthy pregnant women. ${ }^{19,20}$ None of optic ultrasound studies in preeclampsia has, however, compared ONSD neither with direct intracranial pressure measurements nor with radiologic imaging methods. Therefore, all assumptions on intracranial hypertension in severe preeclampsia have to be viewed as such, since they are based on ultrasound findings alone. Moreover, ONSD was not associated with neurological symptoms in neither of these two studies. ${ }^{19,20}$ Regardless of whether increased ONSD in preeclampsia denotes actual increased intracranial pressure, it could be a potential marker of generalized edema. No study to date examined the correlation between ONSD and measures of extravascular fluid in tissues other than the central nervous system. Extravascular fluid in pulmonary interstitium and alveoli is especially concerning in severe preeclampsia, as pulmonary edema continues to be an important cause of maternal morbidity and even mortality. ${ }^{7,10}$ There are no published data on normal numbers of B-lines in anterior intercostal 
spaces in healthy pregnant women. Two studies to date compared lung ultrasound measurements in severe preeclampsia $v s$. healthy pregnant controls. Both used the 28-rib interspaces technique dividing the chest wall in 12 areas on the left and 16 areas on the right anterior and lateral hemithorax. The sum of B-lines in preeclamptic patients was significantly higher than in healthy pregnancies (31 vs. 3 , $\mathrm{p}=0.02$ in the study by Zieleskiewicz et al., and 22 vs. $6, \mathrm{p}=0.002$ in the study by Ambrozic et al.). ${ }^{13,21}$ Numbers of B-lines in healthy pregnancy were, therefore, significantly lower than the median of 19 found in the present study even when posterior lung areas were examined. Our results show that optic ultrasound can help identifying preeclampsia patients with fluid overload in whom additional fluid administration should be avoided and who may even benefit from diuretic therapy.

Despite several advantages of lung ultrasound for identifying excess EVLW, such as its safety in pregnancy by avoidance of ionizing radiation, this technique still requires several measurements and could be time consuming. Optic ultrasound is also a safe and repeatable diagnostic tool, which is even quicker and simpler to perform than lung ultrasound. According to our results it also seems to be effective in diagnosing excess EVLW in patients with severe preeclampsia.

The study has several limitations. One of the main drawbacks is the small study population. The small number of patients included is the result of our decision to focus only on patients with severe preeclampsia. We chose to include only preeclamptic patients with severe features as these are truly critically ill pregnant patients which could mostly benefit from accurate assessment of fluid status. Future research is needed to refute or confirm our findings and to determine whether our results could be generalizable to all patients with preeclampsia, i.e. also to those with milder forms of disease. Future studies are also needed to assess reproducibility of ONSD measurements in everyday clinical practice. Previous publications have reported excellent inter- and intra-observer reproducibility of optic ultrasound as well as steep learning curve of the technique but only within research settings. ${ }^{20,29}$ Another important limitation of the study is its observational nature which does not allow drawing conclusions on prognostic value of ONSD measurements in patients with severe preeclampsia.

In conclusion, ocular ultrasound seems to be a non-invasive and easy-to-learn method for evaluating overall fluid status in patients with severe preeclampsia. It could be especially useful in iden- tifying those preeclamptic patients in which additional fluid intake should be avoided as it could result in pulmonary edema or other complications associated with fluid overload. Further studies are needed to examine the effectiveness of this diagnostic method for reducing maternal complications related to poor fluid management in severe preeclampsia.

\section{References}

1. Ananth CV, Keyes KM, Wapner RJ. Pre-eclampsia rates in the United States, 1980-2010: age-period-cohort analysis. BMJ 2013; 347: f6564. doi: 10.1136/bmj.f6564

2. Hernández-Díaz $\mathrm{S}$, Toh $\mathrm{S}$, Cnattingius $\mathrm{S}$. Risk of pre-eclampsia in first and subsequent pregnancies: prospective cohort study. BMJ 2009; 338: b2255. doi: 10.1136/bmj.b2255

3. Duley L. The global impact of preeclampsia and eclampsia. Semin Perinatol 2009; 33: 130-7. doi: 10.1053/j.semperi.2009.02.010

4. Hutcheon JA, Lisonkova S, Joseph KS. Epidemiology of pre-eclampsia and the other hypertensive disorders of pregnancy. Best Pract Res Clin Obstet Gynaecol 2011; 25: 391-3. doi: 10.1016/j.bpobgyn.2011.01.006

5. Douglas JJ, Walley KR. Fluid choices impact outcome in septic shock. Curr Opin Crit Care 2014; 20: 378-84. doi: 10.1097/MCC.0000000000000116

6. Hayes MA, Timmins AC, Yau EH, Palazzo M, Hinds CJ, Watson D. Elevation of systemic oxygen delivery in the treatment of critically ill patients. N EnglJ Med 1994; 330: 1717-22. doi: 10.1056/NEJM199406163302404

7. Dennis AT, Solnordal CB. Acute pulmonary oedema in pregnant women. Anaesthesia 2012; 67: 646-59. doi: 10.1111/j.1365-2044.2012.07055.x

8. Boyd JH, Forbes J, Nakada TA, Walley KR, Russell JA. Fluid resuscitation in septic shock: a positive fluid balance and elevated central venous pressure are associated with increased mortality. Crit Care Med 2011; 39: 259-65. doi: 10.1097/CCM.0b013e3181feeb15

9. Chaiworapongsa T, Chaemsaithong P, Yeo L, Romero R. Pre-eclampsia. Current understanding of its pathophysiology. Nat Rev Nephrol 2014; 10: 466-80. doi: 10.1038/nrneph

10. Lewis G. The confidential enquiry into maternal and child health (CEMACH). Saving mothers' lives: reviewing maternal deaths to make motherhood safer 2003-2005. The seventh report on confidential enquiries into maternal deaths in the United Kingdom. London: CEMACH; 2007.

11. Royal College of Obstetricians and Gynaecologists. Hypertension in pregnancy. The management of hypertensive disorders during pregnancy. NICE clinical guideline 107. London: FiSH Books; 2011.

12. Brun C, Zieleskiewicz L, Textoris J, Muller L, Bellefleur JP, Antonini F, et al. Prediction of fluid responsiveness in severe preeclamptic patients with oliguria. Intensive Care Med 2013; 39: 593-600. doi: 10.1007/s00134-0122770-2

13. Ambrozic J, Brzan Simenc G, Prokselj K, Tul N, Cvijic M, Lucovnik M. Lung and cardiac ultrasound for hemodynamic monitoring of patients with severe pre-eclampsia. Ultrasound Obstet Gynecol 2017; 49: 104-9. doi: 10.1002/ uog.17331

14. Geeraerts T, Merceron S, Benhamou D, Vigue' B, Duranteau J. Non-invasive assessment of intracranial pressure using ocular sonography in neurocritical care patients. Intensive Care Med 2008; 34: 2062-7. doi: 10.1007/s00134008-1149-x

15. Dubourg J, Javouhey E, Geeraerts T, Messerer M, Kassai B. Ultrasonography of optic nerve sheath diameter for detection of raised intracranial pressure: a systematic review and meta-analysis. Intensive Care Med 2011; 37: 105968. doi: 10.1007/s00134-011-2224-2

16. Loureiro R, Leite CC, Kahhale S, Freire S, Sousa B, Cardoso EF, et al. Diffusion imaging may predict reversible brain lesions in eclampsia and severe preeclampsia: Initial experience. Am J Obstet Gynecol 2003; 189: 1350-5. doi: 10.1067/S0002-9378(03)00651-3 
17. Schwartz RB, Feske SK, Polak JF, DeGirolami U, laia A, Beckner KM, et al. Preeclampsia-eclampsia: clinical and neuroradiographic correlates and insights into the pathogenesis of hypertensive encephalopathy. Radiology 2000; 217: 371-6. doi: 10.1148/radiology.217.2.r00nv44371

18. Zeeman GG, Fleckenstein JL, Twickler DM, Cunningham FG. Cerebral infarction in eclampsia. Am J Obstet Gynecol 2004; 190: 714-20. doi:10.1016/j. ajog.2003.09.015

19. Dubost C, Le Gouez A, Jouffroy V, Roger-Cristoph S, Benhamou D, Mercier FJ, et al. Optic nerve sheath diameter used as ultrasonographic assessment of the incidence of raised intracranial pressure in preeclampsia: a pilot study. Anesthesiology 2012; 116: 1066-71. doi: 10.1097/ALN.0b013e318246ea1a

20. Brzan Simenc G, Ambrozic J, Prokselj K, Tul N, Cvijic M, Mirkovic T, et al. Ocular ultrasonography for diagnosing increased intracranial pressure in patients with severe preeclampsia. Int J Obstet Anesth 2018; [Epub ahead of print]. doi: 10.1016/j.ijoa.2018.06.005

21. Zieleskiewicz L, Contargyris C, Brun C, Touret M, Vellin A, Antonini F, et al. Lung ultrasound predicts interstitial syndrome and hemodynamic profile in parturients with severe preeclampsia. Anesthesiology 2014; 120: 906-14. doi: 10.1097/ALN.0000000000000102

22. American College of Obstetricians and Gynecologists. Task Force on Hypertension in Pregnancy. Hypertension in Pregnancy. Washinton: ACOG; 2013.

23. Geeraerts T, Launey Y, Martin L, Pottecher J, Vigue' B, Duranteau J, et al. Ultrasonography of the optic nerve sheath may be useful for detecting raised intracranial pressure after severe brain injury. Intensive Care Med 2007; 33: 1704-11. doi: 10.1007/s00134-007-0797-6

24. Hansen HC, Helmke K. Validation of the optic nerve sheath response to changing cerebrospinal fluid pressure: Ultrasound findings during intrathecal infusion tests. J Neurosurg 1997; 87: 34-40. doi: 10.3171/ jns.1997.87.1.0034

25. Bäuerle J, Nedelmann M. Sonographic assessment of the optic nerve sheath in idiopathic intracranial hypertension. J Neurol 2011; 258: 2014-9. doi: $10.1007 / \mathrm{s} 00415-011-6059-0$

26. Volpicelli G, Elbarbary M, Blaivas M, Lichtenstein DA, Mathis G, Kirkpatrick AW, et al. International Liaison Committee on Lung Ultrasound (ILC-LUS) for International Consensus Conference on Lung Ultrasound (ICC-LUS): International evidence-based recommendations for point-of-care lung ultrasound. Intensive Care Med 2012; 38: 577-91. doi: 10.1007/s00134012-2513-4

27. Bouhemad B, Mongodi S, Via G, Rouquette I. Ultrasound for "lung monitoring" of ventilated patients. Anesthesiology 2015; 122: 437-47. doi: 10.1097/ ALN.0000000000000558

28. Lichtenstein $D$, Mézière $G$, Biderman $P$, Gepner $A$, Barré $O$. The comet-tail artifact. An ultrasound sign of alveolar-interstitial syndrome. Am J Respir Crit Care Med 1997; 156: 1640-46. doi: 10.1093/eurheartj/ehw164

29. Tayal VS, Neulander M, Norton HJ, Foster T, Saunders T, Blaivas M Emergency department sonographic measurement of optic nerve sheath diameter to detect findings of increased intracranial pressure in adult head injury patients. Ann Emerg Med 2007; 49: 508-14. doi: 10.1016/j.annemergmed.2006.06.040

30. Legrand A, Jeanjean P, Delanghe F, Peltier J, Lecat B, Dupont H. Estimation of optic nerve sheath diameter on an initial brain computed tomography scan can contribute prognostic information in traumatic brain injury patients. Crit Care 2013; 17: R61. doi: 10.1186/cc12589

31. Nabeta HW, Bahr NC, Rhein J, Fossland N, Kiragga AN, Meya DB, et al Accuracy of noninvasive intraocular pressure or optic nerve sheath diameter measurements for predicting elevated intracranial pressure in cryptococcal meningitis. Open Forum Infect Dis 2014; 1: 093. doi: 10.1093/ ofid/ofu093

32. Shirodkar CG, Rao SM, Mutkule DP, Harde YR, Venkategowda PM, Mahesh MU. Optic nerve sheath diameter as a marker for evaluation and prognostication of intracranial pressure in Indian patients: An observational study. Indian J Crit Care Med 2014; 18: 728-34. doi: 10.4103/0972-5229.144015

33. Kim YK, Seo H, Yu J, Hwang GS. Noninvasive estimation of raised intracrania pressure using ocular ultrasonography in liver transplant recipients with acute liver failure -A report of two cases-. Korean J Anesthesiol 2013; 64 : 451-5. doi: 10.4097/kjae.2013.64.5.451
34. Manno E, Motevallian M, Mfochive A, Navarra M. Ultrasonography of the optic nerve sheath suggested elevated intracranial pressure in epilepsy: case report. Internet J Anesthesiol 2013; 26: 262-5. doi: 10.4103/ija.IJA_473_16

35. Fagenholz PJ, Gutman JA, Murray AF, Noble VE, Camargo CA Jr, Harris NS. Optic nerve sheath diameter correlates with the presence and severity of acute mountain sickness: evidence for increased intracranial pressure. J Appl Physiol (1985) 2009; 106: 1207-11. doi: 10.1152/japplphysiol.01188.2007 